\title{
Ueber das chemische Verhalten und die chemische Constitution des Eisenweinsteins;
}

\author{
von \\ H. Wackenroder.
}

Die atomistische Zusammensetzung der weinsteinsauren Doppelsalze ist immer ein Problem gewesen für den Scharfsinn der Chemiker. Man hätte glauben sollen, dafs nach Wa 11 qu ist's u. B ra nde s's Analysen des Brechweinsteins und $D$ ul k's ausführlichen Untersuchungen über die weinsteinsauren Salze (Dissertatio de nonnullis tartratibus 1831; im Auszuge in Schweig ger-Seidel's Journ. für Chemie und Physik, Bd.64.) dieser Gegenstand seine Erledigung gefunden hätte. Gleichwohl sehen wir keine durchgreifende Uebereinstimmung in den Ansichten der Chemilser über diese Salze. Bei Berzelius (Lehrbuch Bd.1V. 1835) finden sich die globuli tartari martiati als weinsaures Kali-Eisenoxydul und der tartarus ferruginosus als weinsaures Kali-Eisenoxyd aufgeführt, während Dulk, wie es scheint, aus seinen analytischen Untersuchungen gefolgert hatte (Journ. für Ch.u.Ph. a.a.O. pag. 213), dafs diese beiden Präparate weinsteinsaures Eisenoxydkali seien, und durch die Formel $4(\mathrm{KO}, \overline{\mathbf{T}})+$ $\left(\mathrm{Fe}_{2} \mathrm{O}_{3} \mathrm{~T}\right)$ repräsentirt werden, dafs aber die von $\mathrm{Gei}$ ger aufgestellte Iypothese, nach welcher der Eisenweinstein aus weinsteinsaurem Eisenoxyd und Eisenoxydkali bestehe, für unbegründet zu halten sei.

Ich habe indessen seit längerer Zeit die Ueberzengung gewomen, dars keine dieser Ansichten ganz richtirg ist, da die globuli tartari martiati eben sowohl, als der tartarus ferruginosus Eisenoxydul und Eisenoxyd zugnleicì enthalten. Man darf der wässrigen Anflösung Arch. d. Pharm. IJ. Rcihe. XXI. Bds. I. Hft. 
der globuli tartari martiati nur etwas Salzsäure und dann theils Kaliumeisencyanid, theils Kaliumeisencyanür hinzufügen, um sich auf der Stelle zu überzeugen, dafs beide Oxyde des Eisens in diesem Präparate zugegen sind. Das quantitative Verhältnifs beider Oxyde mag ein variables sein; dals aber von einer blofs geringfügigen Beimengung des einen oder des andern Oxydes nicht die Rede sein könne, das geht augenscheinlich hervor aus der Menge des Niederschlages von Berlinerblau, welche beide Doppelcyanüre hervorbringen. In der That, es lief's sich auch nichts anderes erwarten, da die Eisenoxydulsalze mit nicht flüchtigen Säuren nur bis zu einem gewissen Grade an der Luft in Eisenoxydsalze übergehen *). Dasselbe Gemenge von beiden Oxyden des Eisens findet sich auch in den globul. tartar. martiat., wclche man mit Hülfe von Salpetersäure leicht bereiten kann. Fügt man nämlich einem mit Wasser angerührten Brei von $10 \mathrm{Grm}$. feiner Eisenfeile und $40 \mathrm{Grm}$. gepulverten gereinigten Weinstein einige Tropfen, im Ganzen 20 bis 25 Tropfen Salpetersäure in längeren Zwischenräumen hinzu, so wird bei gelinder Digestion schon innerhalb ein Paar Tagen ein Eisenweinstein erzeugt, welcher dem gewöhnlichen nur durch langwierige Digestion hervorzubringenden Präparate ganz gleich ist. Während der Digestion verschwindet aus demselben die Salpetersäure bis auf eine unbedeutende Spur. Dafs man übrigens bei Bereitung des Eisenweinsteins die Eisenfeile nicht durch den schwerlöslichen Eisen-

*) Eine wässrige Auflösung des Eisenvitriols wird schon seit 9 Jahren in einer leicht verkorkten Flasche von mir aufbewahrt, und dennoch befindet sich in der iber dem okergelben Bodensatze stehenden dunkelbraunen Flüssigkeit neben dem Eisenoxyd eine gute Menge von Eisenoxydul. 
hammerschlag ersetzen kann, wie Dörffurt vorschlug, ist bekannt genug.

Höchst auffallend ist aber der Umstand, dafs, wie es scheint, ganz dieselbe Mischung von weinsteinsaurem Eisenoxyd, Eisenoxydul und Kali entsteht, wenn Eisenoxydhydrat mit cremor tartari digerirt wird, gemäls der Vorschrift zur Darstellung des tartarus ferruginosus in der Preurs. und Sächs. Pharmakopïe. Bei genauer Befolgung der erwähnten Vorschrift erhält man tartarus ferruginosus an Gewicht $\frac{1}{10}$ mehr, als der angewendete cremor tartari betrug. Es darf jedoch die Digestion des Gemenges aus gepulvertem gereinigtem Weinstein, Eisenoxydhydrat und Wasser nicht länger fortgesetzt werden, als bis die Masse eine weiche Extractconsistenz angenommen hat.

Dieser tartarus ferratus von dunkelgrüner, nach dem Zerreiben von gelbgrüner Farbe, löst sich leicht und völlig klar in kaltem Wasser auf. Die verdünnte Auflösung besitzt eine gelblich-grünliche, die concentrirte eine dunkelgelbgrüne Farbe. Sie röthet das Lackmuspapier stark. - Aetzkali färbt die Auflösung dunkelbraun, beim Erhitzen der Flüssigkeit bis zum Kochen entsteht aber ein voluminöser Niederschlag und das Eisen scheidet sich vollständig ab. War die Auflösung verdünnt, so besteht der Niederschlag fast ganz in Eisenoxydhydrat, war sie aber concentrirt, so ist derselbe von schwarzbrauner Farbe, ähnlich dem durch Fällung hervorgebrachten Aethiops martialis, und besteht aus Eisenoxyd und Eisenoxydul. Die Fällbarkeit des Eisenoxyds aus dem Eisenweinstein durch Kali wird zwar, wie ich glaube, allgemein nicht angenommen; nichtsdestoweniger bernhet diese Annahme auf einem gründlichen Irrthum. - Kohlensaures Natron färbt die Auf- 
lösung grünlich-braun. - Aetzammoniak bringt auch beim Kochen keine Trübung hervor, sondern die Flüssigkeit behält ihre braune Farbe und ihre Klarheit bei. Ein geringer Zusatz von Salzsäure zu. der erwärmten Flüssigkeit bringt jedoch einen Niederschlag von der Farbe des Eisenoxydhydrats hervor, der aber auf Zusatz von mehr Salzsäure verschwindet. Es ist möglich, dafs derselbe basisches weinsteinsaures Eisenoxyd ist, wie D ulk glaubt. - Essigsaures Natron färbt die Flüssigkeit dunkelrothbraun, bewirkt aber auch beim Kochen keine Trübung, selbst wenn eine kleine Menge von Salzsäure hinzugefügt worden. - Phosphorsaures Natron neutralisirt die Auflösung vollkommen, färbt sie dunkelolivengrün, trübt sie jedoch nicht, selbst beim Kochen nicht. Fügt man aber nun noch essigsaures Natron hinzu, so entsteht ein grünlich-weifser Niederschlag und keine Spur von Eisen bleibt in der Flüssigkeit zurück. Versetzt man die Auflösung des tartarus ferratus mit phosphorsaurem Natron und dann mit ein wenig Salzsäure, so schlägt sich besonders beim Erwärmen sogleich ein weifser, voluminöser Niederschlag von phosphorsanrem Eisenoxyd zu Boden, ganz in derselben Weise, wie dieses geschielıt, wenn eine saure Auflösung des phosphorsauren Eisenoxyds mit essigsauren Alkalien vesmischt wird (vergl. dieses Archiv Bd.16. 2. R. pag. 141). Fügt man der Auflösung des tartarus ferratus ziemlich viel phosphorsaures Natron und Salzsäure und dann die nöthige Menge von essigsaurem Natron hinzu, so scheidet sich beim Kochen alles Eisenoxyd als weirses phosphorsaures Eisenoxyd ab, und in der Flüssigkeit bleibt alles Eisenoxydul als phosphorsaures Eisenoxydul aufgelöst. Letzteres ist in der filtrirten Flüssigkeit leicht $\mathrm{zu}$ erkennen, an dem allmälig sich bildenden grünlichen 
Niederschlage durch Actzkali, an dem hellblauen durch Kaliumeisencyanür, an dem Klarbleiben der mit Schwefelwasserstoffwasser vermischten sauren Flüssigkeit, an der geringen rothen Färbung derselben durch Schwefelcyankalium nach vorangegangenem Zusatze von Salzsäure u. s. w. - Kaliumeisencyanid erregt in der Auflösung dieses eisenhaltigen Weinsteins sofort einen starken Niederschlag von Berlinerblau, welcher jedoch auf Zusatz von einigren Tropfen Salzsäure sich noch vermehrt und schneller absetzt, während die Flüssigkeit eine gelbgrünliche Farbe annimmt. - Kaliumeisencyanür bringt nur eine grïne Färbung der Auflösung hervor. Indessen bildet sich auf Zusatz von etwas Salzsäure sogleich ein starker Niederschlag von Berlinerblau, dessen Volumen, nachdem er sich aus der fast farblosen Flüssigkeit abgeschieden hat, ungefähr $\frac{1}{4}$ mehr beträgt, als das Volumen des durch Kaliumeisencyanid hervorgebrachten Berlinerblaues. - Gallusaufgurs färbt die Auflösung dunlselblau und macht sie undurchsichtig. Sie klärt sich auch nicht auf Zusatz von Essigsäure, selbst nicht nach mehren Tagen. - Schwefelwasserstoffwasser erregt in der Auflösung sogleich eine grauschwarze Trübung. Hineingeleitetes Schwefelwasserstoffgas bringt, wie immer, in den Auflösungen des Eisens in organischen Säuren, einen reichlichen schwarzen Niederschlag hervor, jedoch ist, wie ich wenigstens in Bezug auf das essigsaure Eisenoxyd in $B d .16, a .0 . O$. dieses Archivs gezeigt habe, die Fällung des Eisens niemals vollständig.

Aus diesen Reactionen des tartarus ferruginosus geht klärlich hervor, dafs ein guter Theil des Eisenoxyds während der Digestion desselben mit Weinstein zu Oxydul reducirt wird. Dieses kann aber nur auf Kosten per Veinsteinsäure geschehen, und so entsteht die Frage, 
welche Veränderung die Veinsteinsäure dabei erleidet. Es scheint nicht werthlos, dieser muthmafslichen Metamorphose der Weinsteinsäure weiter nachzuforschen. Hr. E der aus Leipzig, welcher aufser mehren anderen Mitgliedern des pharmaceutischen Instituts, vorzüglich Theil hat an diesen Untersuchungen, hat mehre darauf gerichtete Untersuchungen unternommen, deren Resultale im günstigen Falle demnächst mitgetheilt werden sollen.

Obgleich mir längst wohl bekannt war, dafs die globuli tartari martiati im Ganzen dasselbe Verhalten zeigen, wie es von uns jetzt bei dem tartarus ferruginosus gefunden worden, so haben wir dennoch aufs Neue einige vergleichende Versuche angestellt, und zwar sowohl mit frisch bereiteten globulis tartari martiati aus einer Apotheke, als auch mit solchen, die vor $15 \mathrm{Jahren}$ sorgfältig bereitet und während dieser ganzen Zeit in einem nur mit Papier verbundenen Glase als Muster in meiner Sammlung auf́bewahrt wurden. Der Hauptunterschied beruhet nur in dem angewendeten rolhen Weinstein und in der fast unvermeidlichen Beimengung von metallischem Eisen in den Stahlkugeln. Dieses nebst einem grauen schleimigen Rückstand von weinsteinsaurem Kalk hinterbleibt beim Zerreiben der Weinsteineisenkugeln mit kaltem Wasser. War bei der Bereitung der globul. tart. mart. die Digestion mit Eisenfeile nicht lange genug fortgesetzt worden, so bleibt eine grofse Menge metallischen Eisens zurück beim Auflösen des Präparats in Kaltem Wasser, und die Flüssigkeit reagirt stark sauer. Im entgegengesetzten Falle zeigt sie nur eine schwach saure Reaction. Nach dem Volumen der Berlinerblau - Niederschläge zu urtheilen, welche Kaliumeisencyanür und Kaliumeisencyanid in beiden Auf- 
lïsungen hervorbringen, enthält, wie man kaum voraussetzen durfte, das stark saure Präparat ungefähr dieselben Mengen von Eisenoxyd und Eisenoxydul, wie das schwach saure und wie der mit Eisenoxydhydrat bereitete tartarus ferruginosus. Jene ersteren Präparate sind daher dem letztern sehr ähnlich, wo nicht mit demselben identisch. Nur darin, dars die gut gesättigten globuli tartari martiati wenig oder gar Keine freie Säure enthalten und daher mit Kaliumeisencyanid nicht geradezn einen Niederschlag von Berlinerblau geben, dürfte der vorzüglichste Unterschied liegen. Uebrigens verhält sich die wässrige Auflösung der Weinsteineisenkugeln ganz so, wie die Auflösung des tartar. ferruginosus. Beim Kochen mit Aetzkali wird ebenfalls alles Eisen vollkommen niedergeschlagen als Eisenoxyd-OxydulHydrat. Die Flüssigkeit läuft zwar bräunlich durchs Filtrum, allein diese Farbe ruihrt nur von den fremden organischen Theilen aus dem rohen Weinstein her. Diese ertheilen auch der wässrigen Auflösung des Präparats eine dunklere Farbe, als die Auflösung des tartarus ferruginosus besitzt.

Jedenfalls ist es bemerkenswerth, daf's man nach beiden Vorschriften unscrer Pharmakopöen immer ein vierfaches Salz von weinsteinsaurem Eisenoxydul-Kali und weinsteinsaurem Eisenoxyd - Kali, und zwar anscheinend von derselben atomistischen Zusammensetzung erhält. Während in dem einen Falle das entstehende weinsteinsaure Eisenoxydul durch die atmosphärische Luft theilweise zu weinsteinsaurem Eisenoxyd höher oxydirt wird, wird in dem andern Falle das weinsteinsaure Eisenoxyd durch die Weinsteinsäure selbst theilweise $z u$ Eisenoxydulsalz reducirt. 